\title{
1608+656: A QUADRUPLE LENS SYSTEM FOUND IN THE CLASS GRAVITATIONAL LENS SURVEY
}

\author{
STEVEN T. MYERS \\ California Institute of Technology \\ 105-24, Pasadena CA 91125
}

\begin{abstract}
The first phase of a large gravitational lens survey using the Very Large Array at a wavelength of $3.6 \mathrm{~cm}$ has been completed, yielding images for 3258 radio sources. The Cosmic Lens All-Sky Survey, or CLASS, is designed to locate gravitational lens systems consisting of multiply-imaged compact components with separations $>0$ "'2. From this first phase has come the discovery of $1608+656$, a quadruply-imaged object with maximum separation of $2{ }^{\prime \prime} 1$. Images from the Palomar 5-m and Keck 10-m telescopes show the lensed images and the lensing galaxy. An optical spectrum obtained with the Palomar 5-m Telescope indicates a redshift of $z=0.63$ for the lensing galaxy, and a newly-obtained Palomar spectrum indicates a redshift of $z=1.39$ for the lensed source, which appears to be a galaxy. A simple single-galaxy lens model derived from the radio image reproduces the observed configuration and relative fluxes of the images, as well as the position, shape, and orientation of the lensing galaxy. Because a simple mass model is able to fit the observations, we argue that this lens system is promising for determining $H_{0}$. CLASS has also yielded the new double image lens system $1600+434$. The second phase of the survey is scheduled for August and September 1995 on the VLA, and should yield images for an additional $5000+$ targets, bringing the CLASS total to over 8000 .
\end{abstract}

\section{Introduction}

The Cosmic Lens All-Sky Survey (CLASS) is a radio-based survey using the Very Large Array ${ }^{1}$ (VLA) to image approximately 10000 flat-spectrum

\footnotetext{
${ }^{1}$ The National Radio Astronomy Observatory is operated by Associated Universities, Inc., under cooperative agreement with the National Science Foundation.
} 
radio sources, with the aim of finding new gravitational lens systems suitable for cosmographic studies. CLASS is a collaboration between Caltech, Jodrell Bank and Leiden/Dwingeloo (see $§ 5$ ). In many respects, CLASS is an extension of previous VLA surveys such as the Jodrell-Bank/VLA astrometric survey (JVAS: Patnaik et al. 1992). Based on the statistics from JVAS, we expect to find about one lens per 500 sources observed, or 20 new lenses in the total CLASS sample.

Restricting the sample to flat-spectrum sources preferentially selects objects with compact and variable components well-suited to time delay measurements. The exclusion of complex extended steep-spectrum sources from the sample also simplifies the mapping and follow-up tasks. A total of 3258 targets has been observed in the first phase of the survey, CLASS-1. The survey has so far yielded two new lens systems: $1600+434$, a double image system (Jackson et al. 1995) and the quadruple image lens $1608+656$ (Myers et al. 1995), as well as other candidates yet to be followed up.

\section{Observations}

Targets were selected from the $4.85 \mathrm{GHz}$ Green Bank Survey (87GB: Gregory \& Condon 1991). At the time of the CLASS-1 VLA observations, we were able to select 683 sources with two-point spectral indices of $\alpha \geq-0.5$ between the $325 \mathrm{MHz}$ Westerbork Northern Sky Survey (WENSS: de Bruyn et al. 1995) and $4.85 \mathrm{GHz}$, with $4.85 \mathrm{GHz}$ flux densities above $25 \mathrm{mJy}$. The remaining 2575 sources were selected with $\alpha \geq-0.6$ between the $365 \mathrm{MHz}$ Texas Survey (or upon the $4.85 \mathrm{GHz}$ fluxes only in regions not covered by the Texas survey) and $4.85 \mathrm{GHz}$ with $4.85 \mathrm{GHz}$ flux densities above $50 \mathrm{mJy}$. Sources were restricted to galactic latitudes $|b|>10^{\circ}$. CLASS-1 covered the declination range from the northern limit of 87GB $\left(\delta \lesssim 75^{\circ}\right)$ down to $\delta \geq 45^{\circ} 20^{\prime}$ ( $\delta \geq 35^{\circ}$ below the galactic plane).

The CLASS- 1 observations took place during February through May 1994 in the A-configuration of the VLA at a frequency of $8.4 \mathrm{GHz}\left(0^{\prime \prime} \cdot 2\right.$ resolution). For the 2575 non-WENSS sources, two IFs of $25 \mathrm{MHz}$ bandwidth were centered at $8.415 \mathrm{GHz}$ and $8.465 \mathrm{GHz}$. An on-source dwell time of 30 seconds was used. For the WENSS selected sources, the full $50 \mathrm{MHz}$ bandwidth was available due to the accurate WENSS source positions. A phase calibration source from the JVAS was observed every 13.5 minutes. The average target source observation rate was one per minute including overhead from calibration and slewing.

Following initial calibration using AIPS, automatic mapping of the 2575 $87 \mathrm{~GB}$ sources was performed using the DIFMAP package (Shepherd, Pearson \& Taylor, 1994). From the resulting maps $~ 100$ objects with multiple compact components have been selected for further investigation as poten- 
tial gravitational lens candidates. Only a few of these are expected to be real lensed systems. The WENSS-selected sources were analyzed separately (see Jackson et al. 1995), but will be re-analyzed using the automatic mapping for statistical completeness.

\section{The Quad Lens $1608+656$}

The source $1608+656$ (target source $87 \mathrm{~GB} 16087+6540$ ) was observed by CLASS on 1994 March 1. In addition, 1608+656 was observed independently with the VLA in the B-configuration on 1994 July 23, with follow-up observations with the WSRT and OVRO (Snellen et al. 1995). The CLASS VLA data were well fit by a model consisting of four point sources, with no missing extended flux in the residual image. The rms noise level in the final image was $0.44 \mathrm{mJy}$. A total flux density of $73.2 \pm 0.9 \mathrm{mJy}$ was measured, with component flux densities of $35.6,17.8,15.2$, and $4.6 \mathrm{mJy}$ for $\mathrm{A}, \mathrm{B}, \mathrm{C}$ and $\mathrm{D}$ respectively. The brightest component (A) is located at $16^{\mathrm{h}} 09^{\mathrm{m}} 13^{\mathrm{s}} .956+65^{\circ} 32^{\prime} 28^{\prime \prime} \cdot 97$ (J2000), with estimated uncertainty $<0^{\prime \prime} 05$. The WSRT images indicate that $1608+656$ is the lensed core of a large $\left(45^{\prime \prime}\right)$ double radio source (Snellen et al. 1995).

Optical and infrared follow-up observations were undertaken in August and September 1994. An optical image and spectrum of $1608+656$ were obtained with the Palomar Observatory 5 -m Telescope. Approximate photometry on the optical images gives total magnitudes for images and lens of Gunn $r=19.4^{m}$ and $i=19.2^{m}$. A 3000 second exposure using the Double Spectrograph was taken and a redshift of $z=0.6304$ for the lensing galaxy was measured. In the galaxy spectrum, there are strong Balmer absorption lines, indicating that there are A stars present and that the lens might possibly be a post-starburst galaxy.

A $2.2 \mu \mathrm{m}$ ( $\mathrm{K}$ band) infrared image of $1608+656$ was obtained on the W.M. Keck ${ }^{2} 10-\mathrm{m}$ Telescope using the NIRC, in $1^{\prime \prime}$ seeing. The three brightest lens images are easily seen, as is the extended lensing galaxy. The lensing galaxy has a K-band flux roughly four times greater than that of the total emission from the lens components, and has an axial ratio of $b / a=0.56 \pm 0.10$ with its major axis in position angle $60^{\circ}$. Photometry on the lensing galaxy yields a mean surface brightness of $60 \mu \mathrm{Jy} \mathrm{arcsec}{ }^{-2}$ within a circle of radius 1 "' 1 , giving a mean $\mathrm{K}$ surface luminosity of $L_{E}=$ $7.7 \times 10^{8} \mathrm{~L}_{\odot} \mathrm{kpc}^{-2}$.

The CLASS VLA and Keck images, and optical spectrum, are shown in Myers et al.(1995), and are not reproduced here. Figure 1 shows a newly

${ }^{2}$ The W.M. Keck Observatory is operated as a partnership between the California Institute of Technology and the University of California, and was made possible by the generous gift of the W.M. Keck Foundation. 
obtained VLA $5 \mathrm{GHz}$ deep image of $1608+656$. No extended emission is seen to a level of $0.1 \mathrm{mJy}$ at the full resolution of 0 "'33. Also shown is a MERLIN $5 \mathrm{GHz}$ image. The four lensed images are found to be unresolved at the resolution of 50 mas. VLBA observations are scheduled for August 1995, and will show any milli-arcsecond structures that might be associated with the core of a double radio source. Another $8.4 \mathrm{GHz}$ VLA image of $1608+656$ was obtained in July 1995 . There is a significant change in relative fluxes of the components since March 1994: the flux ratio A/B increased by $12 \% \pm 3 \%$ and the ratio $\mathrm{C} / \mathrm{B}$ increased by $36 \% \pm 5 \%$ ( $\mathrm{C}$ became brighter than $\mathrm{B}$ ). There is clearly variability in this object, and therefore a time delay should be measurable.

In late July 1995 , a new spectrum of $1608+656$ was obtained, again with the Double Spectrograph on the Palomar 5-m Telescope. It was possible to identify a feature near $9200 \AA$ previously seen in a Keck LRIS spectrum as a Balmer break, along with the associated Balmer absorption series. A redshift of $z_{s}=1.394$ is derived for the lensed source. The spectrum is indicative of a galaxy, not a quasar as was expected - if true, then the images should be visibly extended in HST observations planned for Cycle 5 .

A simple lens model for $1608+656$ has been constructed using the positions and relative flux densities of the four images in the VLA $8.4 \mathrm{GHz}$ radio map. An oblate spheroidal mass model has been used for the lens, the details of which are given in Myers et al.(1995). A Friedmann-Robertson-Walker universe with a smoothed-out background matter distribution, $q_{0}=0.5$ and $h=H_{0} / 100 \mathrm{~km} \mathrm{~s}^{-1} \mathrm{Mpc}^{-1}$, is assumed. For this model, and a background source redshift of $z_{s}=1.394$, the mass within the equivalent circular Einstein ring radius $\theta_{E}=1^{\prime \prime} 1\left(r_{E}=4.26 \mathrm{kpc}\right)$ is $M_{E}=3.05 \times 10^{11} h^{-1} \mathrm{M}_{\odot}$. Using the previously measured mean $\mathrm{K}$ surface luminosity $L_{E}$, we infer a mean mass-to-light ratio of $M_{E} / L_{E}=6.9 h \mathrm{M}_{\odot} / \mathrm{L}_{\odot}$ inside the cylinder $r_{E}$.

The position and orientation of the lensing galaxy visible in the Keck infrared image agree with those predicted by the model, within the measurement uncertainties. The model axial ratio of $b / a=0.28$ is somewhat lower than the measured 0.56 from the Keck image, although this might be accounted for by the effect of the seeing. The implied model magnifications for images $\mathrm{A}-\mathrm{D}$ plus missing image $\mathrm{E}$ are $+2.58,+1.54,-1.36,-0.35$, and +0.04 respectively (signs denote parity), with a total magnification of 5.87 . According to the model, image B should vary first. The predicted time delays (in $h^{-1}$ days) relative to image B are 40 for A, 44 for $\mathrm{C}$, and 115 for image D. The shortest relative pairwise delay is $4.2 h^{-1}$ days between images $\mathrm{A}$ and $\mathrm{C}$.

The lensing galaxy in the $1608+656$ system presents an interesting puzzle. Whether the lens is a single highly elliptical spheroid, disk galaxy, or a 
pair of close galaxies has yet to be determined. The implied mass within the image radius is large, corresponding to a velocity dispersion of $500 \mathrm{~km} \mathrm{~s}^{-1}$ for an isothermal sphere of core radius $1.9 \mathrm{kpc}$, though the mass-to-light ratio $(7 h)$ is not atypical of other lensing galaxies. This mass is representative of first-ranked cluster ellipticals, while the spectrum and high ellipticity is more suggestive of an early-type spiral galaxy. It is possible that the lens is a close pair of less massive galaxies, explaining the high mass and high ellipticity. The HST observations scheduled for Cycle 5 should provide a better view of the lens and lensed galaxies. This system appears promising for time-delay measurements: it is measurably variable, with known redshifts, a simple mass model (unless it is two galaxies), and four images to provide more constraints on the geometry.

\section{The Future}

The CLASS survey is producing new lenses at around the expected rate of $0.2 \%$. So far, with just over 3200 CLASS- 1 targets observed, there are two confirmed lenses and two further very good candidates. CLASS-2 observations begin in mid-August 1995 and should survey an additional 5000 target sources, and yield another 10 new lenses. When completed, CLASS will yield a carefully selected uniform sample of radio lenses suitable for cosmographic studies. CLASS will also provide a large radio database for investigation of the AGN phenomenon.

Three of the CLASS- 1 candidate lens systems are quads. It appears that just as in other radio-based surveys, quadruple image systems make up on the order of $50 \%$ of lenses. This points to the existence of highly elliptical potentials among the most massive lensing galaxies (eg. King et al. these proceedings).

\section{The CLASS Team}

CLASS is an ambitious undertaking, and is a collaboration between astronomers at Caltech, Jodrell Bank, Leiden, and Dwingeloo observatories. The CLASS team members are:

Caltech S.T. Myers, C.D. Fassnacht, T.J. Pearson, A.C.S. Readhead, M.C. Shepherd (DIFMAP), R.D. Blandford

Jodrell Bank I.W.A. Browne, N. Jackson, T. Muxlow (MERLIN), S. Nair, C. Sykes, P.N. Wilkinson.

Leiden/Dwingeloo A.G. de Bruyn, M. Bremer (WENSS), G.K. Miley, R.T. Schilizzi, I.A.G. Snellen $(1608+656)$

In addition, the optical/IR follow-up of $1608+656$ involved: 
Caltech/Palomar Observatory S.G. Djorgovski, K. Matthews, G. Neugebauer, J.D. Smith, D.J. Thompson, D.S. Womble

\section{References}

de Bruyn, A.G., et al., 1995, in preparation

Gregory, P.C. \& Condon, J.J., 1991, ApJS, 75, 1011

Jackson, N., et al., 1995, MNRAS, 274, L25

King, L. et al., 1995, these proceedings

Myers, S.T., et al., 1995, ApJ (Letters), 447, L5

Patnaik, A.R., Browne, I.W.A., Wilkinson, P.N., \& Wrobel, J.M., 1992, MNRAS, 254, 655

Shepherd, M.C., Pearson,T.J., \& Taylor,G.B., 1994, BAAS, 26, 987

Snellen, I., de Bruyn, A.G., Schilizzi, R.T., Miley, G.K., \& Myers, S.T., 1995, ApJL, 447, L9
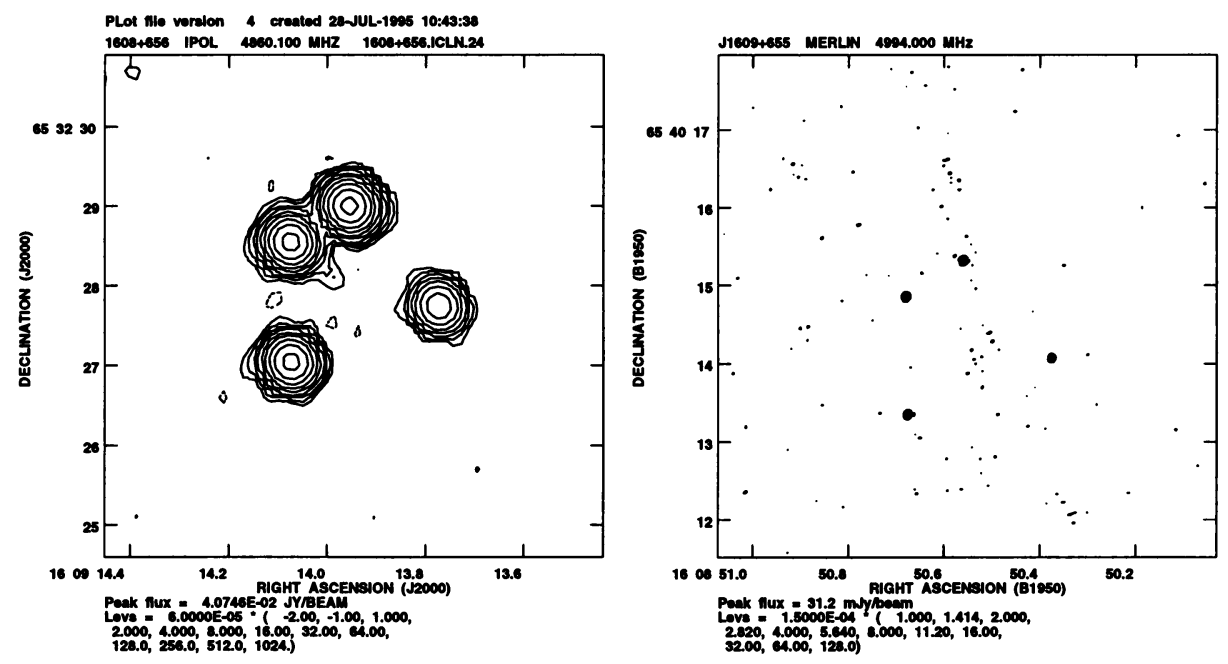

Figure 1. VLA $5 \mathrm{GHz}$ (left), and MERLIN $5 \mathrm{GHz}$ (right) images of 1608+656. Component $\mathrm{A}$ is at the top, $\mathrm{B}$ opposite $\mathrm{A}, \mathrm{C}$ closest to $\mathrm{A}$. 